\title{
Dynamics of Collapse of Air Films in Drop Impact
}

\author{
Jolet de Ruiter, Jung Min Oh, Dirk van den Ende, and Frieder Mugele \\ Physics of Complex Fluids, MESA Institute for Nanotechnology, University of Twente, Post Office Box 217, \\ 7500 AE Enschede, The Netherlands
}

(Received 10 November 2011; published 17 February 2012)

\begin{abstract}
Liquid drops hitting solid surfaces deform substantially under the influence of the ambient air that needs to be squeezed out before the liquid actually touches the solid. Nanometer- and microsecond-resolved dual wavelength interferometry reveals a complex evolution of the interface between the drop and the gas layer underneath. For intermediate impact speeds $(\mathrm{We} \sim 1 \ldots 10)$ the layer thickness can develop one or two local minima-reproduced in numerical calculations - that eventually lead to the nucleation of solidliquid contact at a We-dependent radial position, from a film thickness $>200 \mathrm{~nm}$. Solid-liquid contact spreads at a speed involving capillarity, liquid viscosity and inertia.
\end{abstract}

Liquid drops deform substantially upon impact onto a solid surface. Depending on impact speed they rebound, get deposited on the surface, or disintegrate in a splash (for a review, see Ref. [1]). Following experimental reports of tiny air bubbles being incorporated into drops [2-5] as well as the suppression of splashing upon reducing the ambient air pressure [6] it became clear that the ambient air plays a non-negligible role in the impact process. To describe the expulsion of the air that initially separates the drop from the solid, several models were formulated [7-9] that describe the drop impact primarily in terms of a balance between the inertia of the decelerating liquid and the excess pressure arising from the viscous squeezeout of the thin air layer. A local pressure maximum right under the drop leads to the formation of a "dimple", which should eventually evolve into the enclosed air bubble [9]. Using this model and including corrections due to capillary forces, it was shown that a thin air film of almost constant thickness should develop under the drop [10], and the formation of a thin liquid jet was observed using an axisymmetric, curvilinear description [11]. Mandre et al. [10] suggested that this air film plays an important role, e.g., for the splashing process, but recent experiments by Discroll and Nagel [12] question this scenario: while the presence of interference fringes right under the drop indeed confirms the formation of a dimple, their measurements suggest that direct liquid-solid contact forms very quickly around the dimple, separating dimple from splashing dynamics. Whether air films do play an important role in other regimes of drop impact, how they collapse to establish direct liquid-solid contact, and to what extent the proposed visco-inertial models describe these processes remains unexplored at this stage.

In this letter, we address these issues by monitoring the evolution and the collapse of the air film for a wide range of liquid properties (interfacial tension $\sigma$, viscosity $\mu_{l}$, density $\rho_{l}$ ) at moderate impact speeds. To do so, we develop an advanced high-speed dual wavelength interferometry technique that allows us to extract full thickness profiles with an unprecedented resolution of $\approx 10 \mathrm{~nm}$ and $50 \mu \mathrm{s}$. Focusing on impact speeds of millimeter-sized droplets where both inertial and capillary forces compete with the gas pressure, $\mathrm{We}=R \rho_{l} v^{2} / \sigma \sim 1 \ldots 10$, we demonstrate the transient formation of an air layer with a thickness of a few $100 \mathrm{~nm}$ to a few $\mu \mathrm{m}$ and a life time of a few ms. We identify distinct scenarios for the evolution of the interface profiles, with one or two local minima at different radial positions, for variable impact speed. The visco-inertial model including capillary forces reproduces all salient features including the scaling of the film thickness up to the nucleation of liquid-solid contact. The latter is found to occur within less than $50 \mu \mathrm{s}$ from a distance of 200-500 $\mathrm{nm}$. The subsequent spreading of liquid-solid contact occurs within $\approx 1 \mathrm{~ms}$.

Experiments are done with aqueous droplets, dispensed from a syringe pump to obtain a uniform radius of $R=$ $1.05 \mathrm{~mm}$. We vary the liquid properties using aqueous solutions of ethanol, glycerol, and $\mathrm{CaCl}_{2}$, and obtain the following properties: $\sigma=27-65 \mathrm{mN} \cdot \mathrm{m}^{-1}, \mu_{l}=$ $1-109 \mathrm{mPa} \cdot \mathrm{s}$, and $\rho_{l}=997-1366 \mathrm{~kg} \cdot \mathrm{m}^{-3}$. The droplets are impacting with a velocity $v$ between 0.11 and $0.53 \mathrm{~m} \cdot \mathrm{s}^{-1}$ onto carefully cleaned glass substrates (cover slip) with an rms surface roughness below $2 \mathrm{~nm}$ (measured with atomic force microscopy). Low concentrations of dye (rhodamin and fluorescein) are added to the liquids to suppress internal light reflections within the drop. The approach and impact are imaged in reflection mode through the transparent glass substrate by dual wavelength reflection interference microscopy (DW-RIM) at the 431 and $550 \mathrm{~nm}$ spectral lines (each with a bandwidth of $\sim 30 \mathrm{~nm}$ ) of a mercury lamp. The separate interference signals are recorded with two synchronized high-speed cameras (Photron SA3 and SA5) at recording speeds up to $20000 \mathrm{fps}$ (which limits the We number of the impact). Measurements that do not require absolute film thickness measurements (such as the spreading of solid-liquid 
contact) are possible up to 75000 fps. Figure 1(a) and movie S1 [13] show typical interference patterns of the squeezed air film (for the primary wavelength, $550 \mathrm{~nm}$ ). The nonmonotonic spacing of the interference fringes indicates a rather complex thickness profile of the air layer with several inflection points. We extract absolute thickness profiles [see Fig. 1(d)] with a maximum accuracy of $\approx 10 \mathrm{~nm}$, by combining the information of the two wavelengths [14] and by interpolating between local maxima and minima of the intensity. Our optical model for the glass-air-water system shown in Fig. 1(c) $[13,15]$ takes into account the finite aperture of the optics, the bandwidth of the light, as well as the spectral emission and sensitivity of the lamp and the cameras, respectively [16,17].

We first analyze the time evolution of the liquid-air interface before liquid-solid contact (see Fig. 2). When the drops enter our depth of view, they are already deformed and display a characteristic dimple profile with a
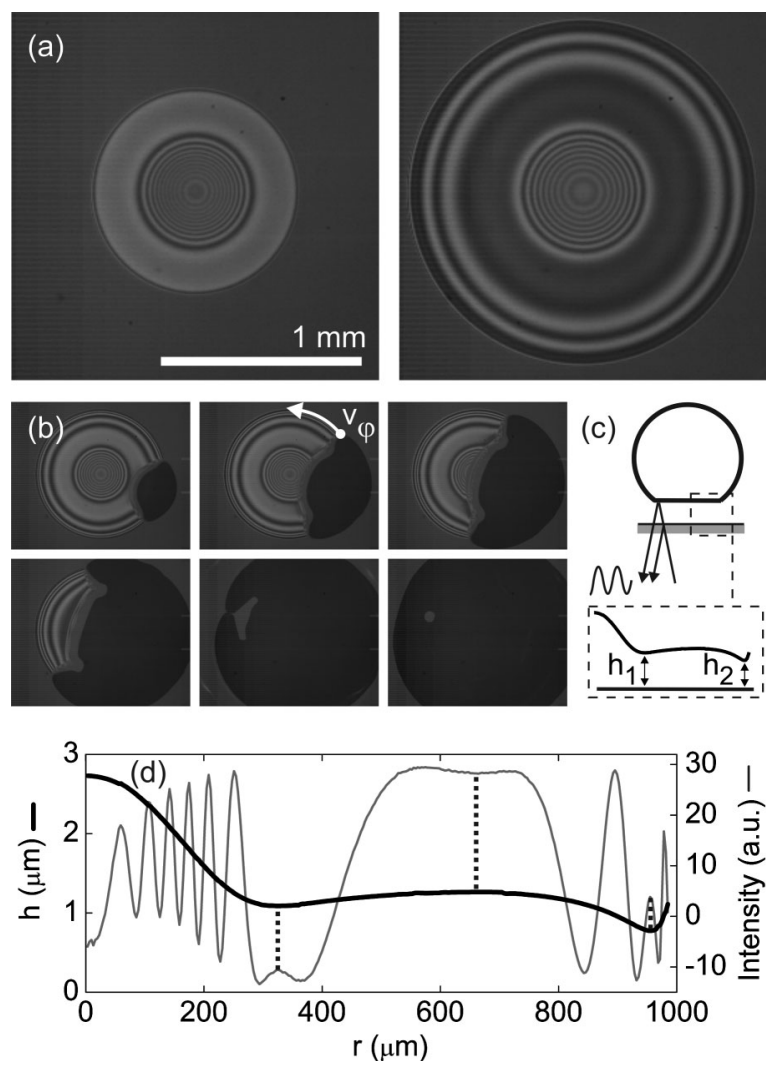

FIG. 1. Droplet cushioning, and spreading of liquid-solid contact at moderate We impact. (a) Interference profile of the air film below a water droplet at (left) 4.1 and (right) $2.3 \mathrm{~ms}$ before nucleation of a liquid-solid contact. (b) Spreading of liquid-solid contact at $0.10,0.21,0.32,0.49,0.75,1.05 \mathrm{~ms}$ after nucleation. $v_{\varphi}$ : azimuthal spreading velocity. (c) Sketch of deformed drop indicating interference of light reflected from the solid/air and air/liquid interfaces. Inset: schematic dimple with local minima $h_{1}$ and $h_{2}$. (d) Characteristic radial intensity profile (thin grey line), and the corresponding interface profile (thick black line). Dotted lines indicate positions of local extrema in $h$. central height of 4-5 $\mu \mathrm{m}$. A "kink" of high local curvature at height $h_{1}$ marks the edge $r_{1}$ of the dimple. As time proceeds, this kink first approaches the substrate along with the central dimple. At some $v$-dependent value, both $h_{1}$ and $r_{1}$ saturate. As the drop spreads further, an air layer of approximately constant thickness is entrapped. For low impact speeds, see top profiles in Fig. 2, the thickness of this layer eventually decreases and develops a second even sharper kink at $r_{2}$ as the drop approaches its maximum extension. The thickness $h_{2}$ at $r_{2}$ decreases linearly with time-with a velocity which is 3 orders of magnitude smaller than the initial drop velocity-, see Fig. 3(b), and eventually leads to the nucleation of liquid-solid contact. During this entire process, the film profile including $h_{1}$ remains essentially invariant in the range of $r \approx 200 \ldots 700 \mu \mathrm{m}$. For somewhat higher impact speeds, see middle profiles in Fig. 2, the overall thickness of the air layer decreases, and it develops a slight slope of $\approx 0.1^{\circ}$. Finally, a second kink develops and induces liquid-solid contact. For even higher speeds, see bottom profile in Fig. 2, however, no second kink is observed. Rather, $h_{1}$ becomes so small that nucleation of liquid-solid contact is

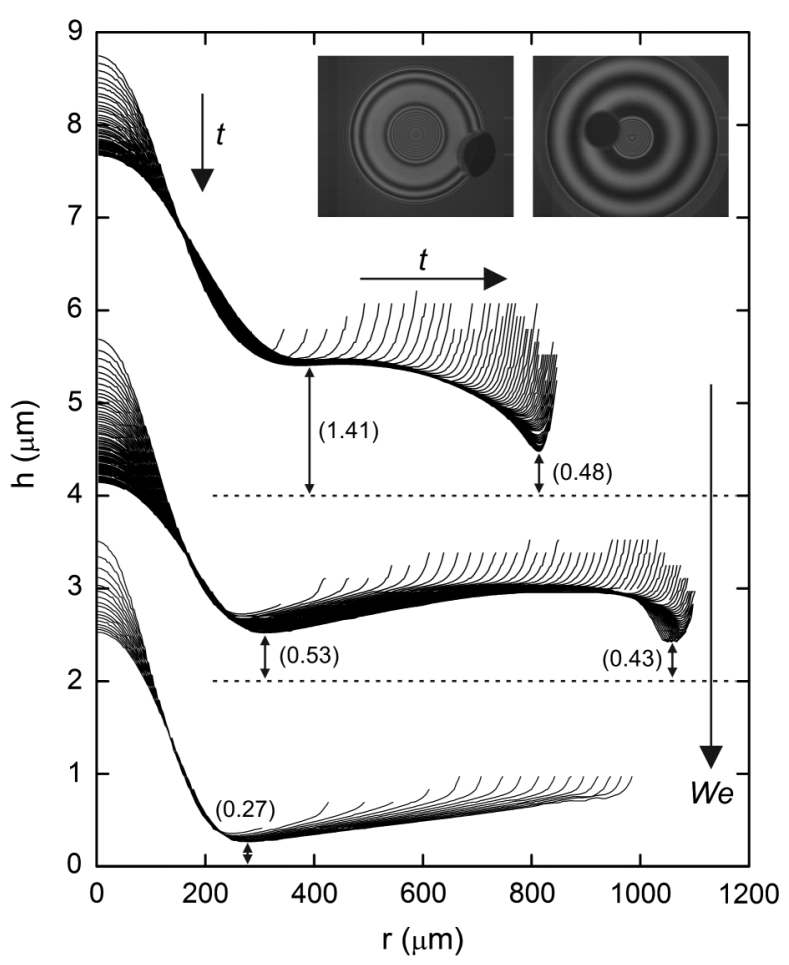

FIG. 2. Time evolution of interface profiles for water drops with increasing impact velocities $v$ (top to bottom: 0.24, 0.41, $0.52 \mathrm{~m} \cdot \mathrm{s}^{-1}$; corresponding We numbers: 0.9, 2.6, 4.2). Time step: $50 \mu \mathrm{s}$. Profiles for different $v$ are shifted vertically for clarity. Note the different scales on the abscissa and the ordinate: maximum slope of interface $<3^{\circ}$. Numbers in parentheses indicate minimum heights in $\mu \mathrm{m}$ of $h_{1}$ and $h_{2}$. Inset: nucleation of solid-liquid contact at $r_{2}$ and $r_{1}$ for low (left) and high (right) We, respectively. 

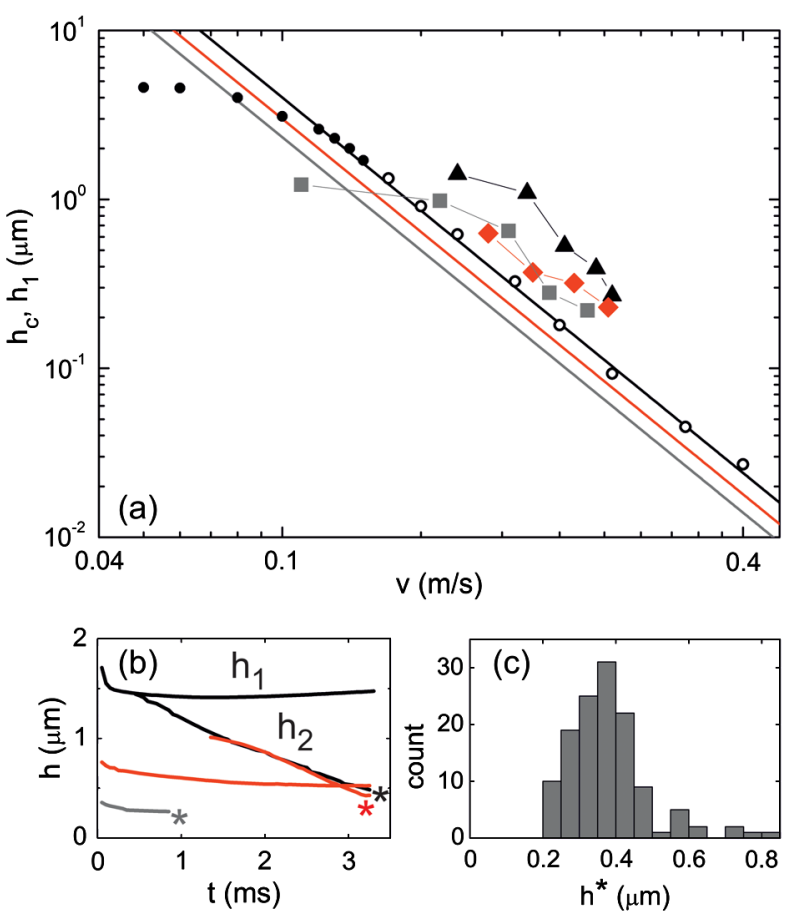

FIG. 3 (color online). Temporal evolution of local minima, and their equilibrium and nucleation height. (a) Symbols: $h_{1}$ versus impact velocity $v$ for experiments with water (black triangles), ethanol (red diamonds), and $\mathrm{CaCl}_{2}$ (grey squares) drops. Solid lines: universal scaling law for $h_{c}$ (colors according to experimental conditions). Dots: numerical values of $h_{1}$ for water; open dots: profiles with one local minimum, solid dots: two local minima. (b) $h_{1}$ and $h_{2}$ vs time for the experiments shown in Fig. 2. From top to bottom: $v=0.24,0.41$, and $0.52 \mathrm{~m} \cdot \mathrm{s}^{-1}$. The star indicates which of the two minima nucleates-at a height $h^{*}$. (c) Histogram showing the value of $h^{*}$ in 128 experiments with various fluid properties (bin size $50 \mathrm{~nm}$ ).

observed before the drop reaches its maximum lateral extension; see inset of Fig. 2. This transition from a twokink scenario at low impact speeds to a single-kink scenario at higher $v$ with liquid-solid contact nucleation at $r_{2}$ and at $r_{1}$, respectively, is consistently observed for all liquids. It occurs at $\mathrm{We} \approx 1$ suggesting an important role of capillary forces in suppressing the kink at $r_{1}$ for low We.

These results confirm the formation and subsequent evolution of the dimple including the existence of a thin transient air film of approximately constant submicrometer thickness, as predicted by the visco-inertial model including capillary forces $[8,10]$. To achieve a more detailed comparison we calculated surface profiles for a wide range of We using this model. Indeed, we find transition from a regime involving two local minima to one local minimum around $\mathrm{We} \approx 1$, see Fig. S2 [13]. From these calculations, we extract a numerical value of $h_{1}$ as a function of $v$; see Fig. 3(a). For We $>0.23$ (here: $v>0.12 \mathrm{~m} \cdot \mathrm{s}^{-1}$ ), $h_{1}$ follows the universal scaling law for the crossover height $h_{c}=2.54 R \mathrm{St}^{14 / 9} \mathrm{Ca}^{-2 / 3}$ at which capillary forces exceed the initially dominant gas pressure as resisting force at the location of the (first) kink due to the increasing local curvature [10]. Here the Stokes number $\mathrm{St}=\mu_{g} /\left(\rho_{l} v R\right)$ gives the ratio of viscous forces in the gas to inertial forces in the drop, and $\mathrm{Ca}=\mu_{g} v / \sigma$ is the capillary number in the gas (with $\mu_{g}$ the gas viscosity). For We $<0.23$, both the capillary pressure and the gas pressure remain low and in balance throughout the entire impact process. As a result, $h_{1}$ falls below $h_{c}$. Our experimental data agree with these predictions [see symbols in Fig. 3(a)] - yet two aspects are remarkable. (i) The absolute value of $h_{1}$ exceeds the expectation by a factor $\approx 3$. At first glance one might worry that the two-dimensional character of the calculations as compared to the three-dimensional experiments might cause this deviation. However, the physical processes controlling the value of $h_{c}$ occur at lateral scales much smaller than the drop size and should therefore not be affected by the macroscopic geometry. Yet, this deviation is in line with a more recent implementation of the theory [18], which states that the originally reported prefactor in the scaling law for $h_{c}$ is too small. According to that work the actual value should be 5 , which is close to but still somewhat below our experimental finding. (ii) We never find values of $h_{1}$ (nor $h_{2}$ ) below $200 \mathrm{~nm}$. Typically for $\mathrm{We} \sim 1 \ldots 10$, liquid-solid contact can nucleate at any random moment within $50 \mu \mathrm{s}$ as soon as either $h_{1}$ or $h_{2}$ decreases below $\approx 500 \mathrm{~nm}$. Figure 3(c) shows a histogram of the "nucleation height" $h^{*}$ for a large number of drops. We find an average value of $h^{*} \approx 370 \mathrm{~nm}$. This value is remarkably large. Extensive AFM imaging of our glass surfaces shows that this value exceeds by far the roughness of our surfaces. At the same time, molecular forces such as attractive van der Waals forces between the liquid and the solid are negligible at these distances. We note, however, that $h^{*}$ is only a few times the mean free path in the gas at ambient pressure. (Our numerical calculations indicate that the local gas density is not substantially enhanced for our experimental conditions.) At such distances rarefied gas effects begin to reduce the gas pressure $[11,19,20]$ and thereby might promote the collapse of the air layer. Extrapolating to higher impact speeds, this suggests that the thin air layers predicted for the splashing regime [10] are probably unstable, and hence not responsible for splashing-in agreement with the recent results by Driscoll and Nagel [12].

Finally, we analyze the spreading behavior of liquidsolid contact following nucleation. As shown in Fig. 1(b), the liquid-solid contact area preferentially spreads in the azimuthal direction along the ring of minimum gas layer thickness, forming two characteristic cusps. The azimuthal spreading speed $v_{\varphi}$ turns out to be constant in time, see Fig. 4(a), with values of 0.2 to $4 \mathrm{~m} / \mathrm{s}$, depending on $h^{*}$ as well as the properties of the fluid. If we neglect the effects of liquid viscosity, both Bernoulli's equation and conservation of momentum with $v_{\varphi}$ the only dominant velocity component, $\rho_{l} \partial_{t} v_{\varphi}+\partial_{x} p=0$, with $p \propto \sigma / h^{*}$ yield the 

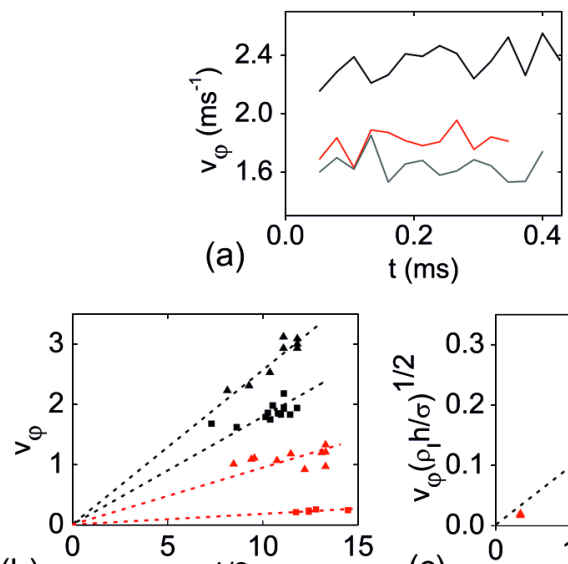

(b)

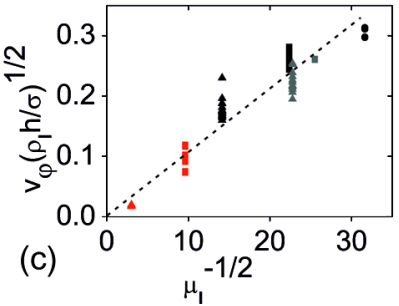

FIG. 4 (color online). Spreading of liquid-solid contact. (a) Azimuthal velocity $v_{\varphi}$ for $\mathrm{CaCl}_{2}$ droplets with $h_{1}=0.23$, 0.57 , and $0.74 \mu \mathrm{m}$ (from top to bottom). (b) $v_{\varphi}$ versus the inertial scaling for increasing viscosity (top to bottom). (c) $v_{\varphi}$, nondimensionalised by the inertial scaling, versus $\mu_{l}^{-1 / 2}$ for experiments with seven fluids having viscosities ranging from $1 \ldots 10^{2} \mathrm{mPa} \cdot \mathrm{s}$. (The error in the determination of $v_{\varphi}$ is typically $5 \%$. All variables given in SI units.)

scaling $v_{\varphi} \propto\left(\sigma / \rho_{l} h^{*}\right)^{1 / 2}$ that is independent of time. This is consistent with the capillarity-inertia controlled regime of drop spreading described earlier by Biance et al. [21] if adapted to the one-dimensional situation of our azimuthal spreading process. This inertial scaling was also found to be valid in the partial wetting regime when including a dependence on equilibrium contact angle [22]. Yet, this inertial description is incomplete. For aqueous drops of variable glycerol content, we find a clear decrease of $v_{\varphi}$ with increasing viscosity; see Fig. 4(b). Empirically, we find that the normalized azimuthal velocities for variable viscosity collapse if plotted vs $\mu_{l}^{-1 / 2}$; see Fig. 4(c). We do currently not have any explanation for this behavior. Possible approaches to include the effect of the liquid viscosity include the growth of a viscous boundary layer from the solid substrate into the accelerating liquid as well as viscous friction in the vicinity of the moving three phase contact line during spreading [23]. The latter would introduce a dynamic contact angle that modifies the shape of the liquid-vapor interface around the contact area. Our results suggest that a similar viscosity dependence would play a role in spreading experiments such as described in Refs. [21,22].

In summary, high-speed dual wavelength interferometry provides the first experimental characterization of the evolution of the theoretically predicted gas layer and its role in drop impact. Our measurements reveal the formation of a dimple right under the impacting drop, which can be surrounded by a thin air layer depending on impact speeds. Nucleation of liquid-solid contact occurs from a surprisingly large distance of $>200 \mathrm{~nm}$. The detailed time evolution of the interface provided here will enable quantitative numerical studies to elucidate the role of rarefied gas effects for this process as well as the influence of viscous dissipation during the subsequent spreading of liquid-solid contact.

We thank Chandra Murade for valuable help with the optical technique. We thank HIPRINS for supporting this work. This project is co-sponsored by the Dutch Ministry of Economic Affairs, Agriculture and Innovation, the provinces Overijssel, Limburg, and Noord-Brabant, and cityregion Eindhoven (SRE).

[1] A. L. Yarin, Annu. Rev. Fluid Mech. 38, 159 (2006).

[2] H. Fujimoto, H. Shiraishi, and N. Hatta, Int. J. Heat Mass Transfer 43, 1673 (2000).

[3] V. Mehdi-Nejad, J. Mostaghimi, and S. Chandra, Phys. Fluids 15, 173 (2003).

[4] S. T. Thoroddsen, T. G. Etoh, and K. Takehara, J. Fluid Mech. 478, 125 (2003).

[5] D. van Dam and C. Le Clerc, Phys. Fluids 16, 3403 (2004).

[6] L. Xu, W. W. Zhang, and S. R. Nagel, Phys. Rev. Lett. 94, 184505 (2005).

[7] F. T. Smith, L. Li, and G. X. Wu, J. Fluid Mech. 482, 291 (2003).

[8] M. Mani, S. Mandre, and M. P. Brenner, J. Fluid Mech. 647, 163 (2010).

[9] P. D. Hicks and R. Purvis, J. Fluid Mech. 649, 135 (2010).

[10] S. Mandre, M. Mani, and M. P. Brenner, Phys. Rev. Lett. 102, 134502 (2009).

[11] L. Duchemin and C. Josserand, Phys. Fluids 23, 091701 (2011).

[12] M. M. Driscoll and S.R. Nagel, Phys. Rev. Lett. 107, 154502 (2011).

[13] See Supplemental Material at http://link.aps.org/ supplemental/10.1103/PhysRevLett.108.074505 for details.

[14] J. Schilling, K. Sengupta, S. Goennenwein, A. R. Bausch, and E. Sackmann, Phys. Rev. E 69, 021901 (2004).

[15] J. de Ruiter, D. van den Ende, and F. Mugele (to be published).

[16] O. Theodoly, Z.H. Huang, and M.P. Valignat, Langmuir 26, 1940 (2010).

[17] T. Becker and F. Mugele, J. Phys. Condens. Matter 17, S319 (2005)

[18] S. Mandre and M. P. Brenner, J. Fluid Mech. 690, 148 (2011).

[19] P. Zhang and C. K. Law, Phys. Fluids 23, 042102 (2011).

[20] A. Gopinath, S. B. Chen, and D. L. Koch, J. Fluid Mech. 344, 245 (1997).

[21] A.-L. Biance, C. Clanet, and D. Quéré, Phys. Rev. E 69, 016301 (2004).

[22] J. C. Bird, S. Mandre, and H. A. Stone, Phys. Rev. Lett. 100, 234501 (2008).

[23] O. V. Voinov, Fluid Dyn. 11, 714 (1977). 